\title{
A Logic for Probabilities in Semantics
}

\author{
M. Andrew Moshier* Achim Jung ${ }^{\dagger}$
}

September 9, 2003

\begin{abstract}
Probabilistic computation has proven to be a challenging and interesting area of research, both from the theoretical perspective of denotational semantics and the practical perspective of reasoning about probabilistic algorithms. On the theoretical side, the probabilistic powerdomain of Jones and Plotkin represents a significant advance. Further work, especially by Alvarez-Manilla, has greatly improved our understanding of the probabilistic powerdomain, and has helped clarify its relation to classical measure and integration theory. On the practical side, many researchers such as Kozen, Segala, Desharnais, and Kwiatkowska, among others, study problems of verification for probabilistic computation by defining various suitable logics for the classes of processes under study. The work reported here begins to bridge the gap between the domain theoretic and verification (model checking) perspectives on probabilistic computation by exhibiting sound and complete logics for probabilistic powerdomains that arise directly from given logics for the underlying domains.

The category in which the construction is carried out generalizes Scott's Information Systems by taking account of full classical sequents. Via Stone duality, following Abramsky's Domain Theory in Logical Form, all known interesting categories of domains are embedded as subcategories. So the results reported here properly generalize similar constructions on specific categories of domains. The category offers a promising universe of semantic domains characterized by a very rich structure and good preservation properties of standard constructions. Furthermore, because the logical constructions make use of full classical sequents, the morphisms have a natural non-deterministic interpretation. Thus the category is a natural one in which to investigate the relationship between probabilistic and non-deterministic computation. We discuss the problem of integrating probabilistic and non-deterministic computation after presenting the construction of logics for probabilistic powerdomains.
\end{abstract}

\section{Introduction}

The probabilistic powerdomain construction of Jones and Plotkin [JP89, Jon90] has proved to have applications beyond its origins as a tool for modelling probabilistic algorithms within domain theory. Edalat [Eda95] employs the probabilistic powerdomain construction toward the

${ }^{*}$ M. Andrew Moshier (Communicating author), Department of Mathematics, Computer Science and Physics, Chapman University, Orange, CA 92867, USA. Phone +1 714997 6628, Fax +1 714532 6048, Email moshier@chapman. edu.

${ }^{\dagger}$ School of Computer Science, The University of Birmingham, Edgbaston, Birmingham, B15 2TT, England. Email A. Jung@es. bham. ac.uk 
study of fractals within a domain theoretic framework. Desharnais, et al [DEP98, DEP97, DEPar] study problems of verification for labelled Markov processes. And closer to the construction's origins, Mislove [Mis00] and Tix [Tix99] investigate how to integrate nondeterministic choice and probabilistic algorithms smoothly. McIver [McI01] looks at a similar problem from a more applied perspective.

The work of Desharnais, et al, McIver, as well as Morgan, et al, [MMS96] are of particular interest to us because they involve the development of logics for reasoning about various probabilistic phenomena (such as labelled Markov processes). They suggest that a uniform treatment of how such logics may arise will prove to be useful. In this work, we provide such a treatment, showing how to construct a logical description of the probabilistic powerspace for any stably compact topological space.

Let us explain this last statement in some more detail. At the heart of our approach is an equivalence between (logical) theories and (denotational) models. On the logical side this means that we work with sets of axioms about concrete propositions and universally valid inference rules. On the semantic side we exhibit the structures which can be characterised by a logical theory. The classical example of such a correspondence is the Stone Representation Theorem: Every propositional theory corresponds uniquely to a totally disconnected compact Hausdorff space. The insight, that Stone duality can be used to link denotational semantics and program logics, is due to Smyth. It forms the basis of Abramsky's Domain Theory in Logical Form and was put to work in two substantial case studies, [Abr91, Abr90]. Abramsky does not work with full propositional logic and Stone spaces but, rather, he drops negation and implication, and employs the equivalence between theories of the remaining positive propositional logic and spectral spaces (which encompass all classical semantic domains, such as Scott-domains or bifinite domains). The class of spectral spaces, however, does not contain continuous spaces, such as the unit interval, and it is therefore not surprising that the setting needs to be further expanded in order to accommodate probabilities. Indeed, our work [JKM99] is based on a further weakening of the logic by dropping the reflexivity axiom $(\phi \Vdash \phi)$ and by the correspondence between theories in this non-reflexive positive logic and stably compact spaces.

This paper stresses the logical side of this correspondence and it is not necessary to be an expert in the topological properties of stably compact spaces in order to appreciate the results reported below. We will summarize the key properties in Section 2 and the reader interested in a fuller story should consult [JS96, Keg99] or the forthcoming [GHK ${ }^{+}$02]. For our present purposes it is sufficient to recall a crucial result in the thesis of Alvarez-Manilla [AM01], where the category SCS stably compact spaces is shown to be closed under the probabilistic powerspace construction. The only other closure results for this construction concern dcpo's (trivially), continuous domains [JP89, Jon90], and Lawson-compact continuous domains [JT98], but unlike SCS neither of these categories has a good logical description (via Stone duality, as explained above) nor many other closure properties as one needs for building a denotational semantics.

The logic, as we have said before, is propositional logic restricted to conjunction and disjunction (including the nullary versions, true and false), and reflexivity is not assumed. This has the consequence that a Hilbert-style presentation, to the best of our knowledge, is not possible, and that instead Gentzen-style sequents

$$
\phi_{1}, \ldots, \phi_{n} \Vdash \psi_{1}, \ldots, \psi_{m}
$$

become the basic syntactic unit (specifically, $\Vdash$ is part of the object syntax, as in Gentzen's 
sequent calculus, and not a meta-symbol denoting provability). The logic was first presented in [JKM97] and [JKM99], but it builds on the earlier [Smy92a, JS96] and in essence is an elaboration of Abramsky's Domain Theory in Logical Form for continuous spaces. It is shown in [JKM99] that despite non-reflexivity some standard proof-theoretic concepts, such as cut elimination, still apply.

Under Stone duality, a proposition $\phi$ corresponds to an open set $o \llbracket \phi \rrbracket$; it was argued by Smyth [Smy83, Vic89, Smy92b] that this is in order: open sets correspond to semi-decidable properties and these are precisely the ones which ought to be of relevance in program logics. In our setting, we observe that furthermore, a sequent $\Gamma \Vdash \Delta$ translates to a "strong containment" $o \llbracket \Gamma \rrbracket \Subset o \llbracket \Delta \rrbracket$ of open sets which is itself "observable" or "semi-decidable". However, we hasten to add that in the presence of non-determinism or probabilistic choice, the label "observable" has to be taken with a grain of salt.

From a motivational point of view, the language of "observable properties" is, however, useful for choosing the right primitives for a probabilistic logic. On the spatial side it is thus natural to consider maps $v$ which assign a probability to all open subsets, and which have the following properties:

1. [Continuity] For directed sets $\left\{U_{i}\right\}_{i}$ of opens, $v\left(\bigcup_{i} U_{i}\right)=\sup _{i}\left\{v\left(U_{i}\right)\right\}$.

2. [Strictness] $v(\emptyset)=0$.

3. [Modularity] For all opens $U$ and $V, v(U)+v(V)=v(U \cap V)+v(U \cup V)$.

4. [Normalcy] $v(X)=1$.

We call such functions probability valuations (or sub-probability valuations if (4) is replaced by $v(X) \leq 1$ ). They were first introduced into denotational semantics by the seminal work of Jones and Plotkin [JP89, Jon90], whereas earlier work, e.g. by Kozen [Koz81], employed measures. The exact connection between valuations and measures has always been of interest in Mathematics, we only mention [SD80, Law82, AMESD00] and refer to [AM01] for a comprehensive treatment. For us it is reassuring to know that on stably compact spaces, probability valuations extend uniquely to Radon measures and every Radon probability measure arises in this way. More importantly for us, Alvarez-Manilla shows that the set of (normal) valuations over a stably compact space can be given a stably compact topology that lies between the Scott topology and the topology of weak convergence. This opens the prospect that this probabilistic powerspace can be described logically. But even better, we now know [AMJK] that the topology is actually equal to the weak topology (which is generally finer than the Scott topology). This is of relevance because it shows that Alvarez-Manilla's topology is precisely the weakest topology to make the integral $v \mapsto \int f d v$ a (Scott) continuous operation for every semi-continuous real-valued $f$. As one then easily infers, the canonical subbasic opens for the weak topology are the sets $O_{q}:=\{v \in \mathrm{V}(X) \mid v(O)>q\}$ for $O$ open in $X, q$ a rational number between 0 and 1 . In our probabilistic logic we should therefore analogously work with basic propositions $\phi_{q}$, interpreted as "proposition $\phi$ holds with probability greater than $q$ ". This is indeed the approach that we shall take.

In order to complete this programme, one needs to find the proof rules for entailments between propositions of this shape and show soundness and completeness with respect to the intended space of all probability valuations. The situation becomes clearer by using a modicum of categorical terminology. The stably compact spaces introduced above form a subcategory SCS of the category Top of topological spaces and continuous functions. Also 
of interest is the category SCS* where the objects are the same but morphisms are closed relations (see Section 2 below for details). SCS can be identified with a subcategory of SCS*. On the logical side, every theory in our non-reflexive propositional logic is an object in the category MLS, where morphisms between theories are entailment relations $\vdash$ very similar to the internal reasoning in a theory. The key result of [JKM99] is that SCS* and MLS are equivalent. This equivalence cuts down to one between SCS and MLS ${ }^{f}$, where the entailment relations satisfy an additional property.

In order more fully to exploit this equivalence between semantics and logic, one then strives to lift it to constructions, that is, given a construction $T$ (possibly in several variables) on SCS*, one seeks a "logical" construction $\bar{T}$ which respects the equivalence:

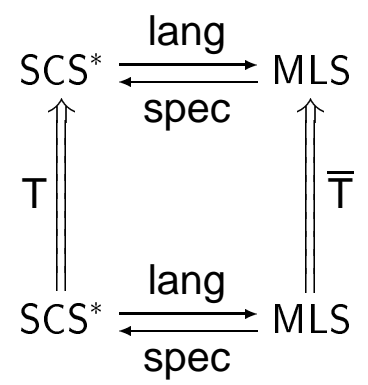

Generally, $\overline{\mathrm{T}}$ is defined via proof rules, and the commutativity of the above diagram is shown by establishing lang $\circ \mathrm{T} \approx \overline{\mathrm{T}} \circ$ lang.

For the probabilistic powerspace the task is to difficult to accomplish in one go. Our proof of completeness therefore borrows an idea of Reinhold Heckmann's [Hec94] and, like him, we carry out the construction in four stages. This produces logical descriptions for all of the following:

- $\mathrm{C} \Omega(X)$, the space of Scott continuous functions from $\Omega(X)$ to $[0,1]$ with the compactopen topology (which coincides with both the weak and the Scott-topology);

- $\mathrm{C}_{s} \Omega(X)$, the subspace of $\mathrm{C} \Omega(X)$ consisting of strict continuous functions;

- $\mathrm{V}(X)$, the subspace of $\mathrm{C}_{s} \Omega(X)$ consisting of modular strict continuous functions, i.e., valuations;

- $\mathrm{V}^{1}(X)$, the subspace of $\mathrm{V}(X)$ consisting of normal valuations.

\section{Stably Compact Spaces}

A subset of a topological space $X$ is saturated if and only if it is an intersection of opens. In particular, every open is saturated and the saturation of a subset is the intersection of its neighborhood filter. A subset is compact if and only if its saturation is compact. Compact saturated sets play a key role in our setting.

Definition 2.1. A topological space is called stably compact if it is sober, locally compact and stable (i.e., finite sets of compact saturated subsets have compact intersection).

We insist on sobriety because our general framework is Stone duality and we want to represent spaces by (sublattices of) their frame of opens (which we interpret as extensions of 
logical propositions). In contrast to Geometric Logic, we axiomatize the way-below relation between open sets, rather than inclusion. Local compactness is precisely the condition which guarantees that the former is rich enough to reconstruct the latter. Stability, finally, is convenient because it allows us to deal with opens and compacts in the same logical framework.

Examples of stably compact spaces include various classes of domains in their Scotttopologies, such as continuous lattices, Scott-domains, bifinite domains, and FS-domains. Also included are all compact Hausdorff spaces.

We denote the specialization order on $X$ by $\leq_{X}$. Unlike with domains, the topology of a stably compact space in general cannot be reconstructed from the order alone.

We denote with $\Omega(X)$ the frame of open sets (ordered by inclusion) and with $\mathcal{K}(X)$ the lattice of compact saturated sets ordered by reversed inclusion. For a stably compact space both are continuous distributive lattices, in particular, $\mathcal{K}(X)$ is the set of closed sets for a topology on $X$, called the co-compact topology. We denote the resulting space by $X_{\kappa}$. From what we have said before it follows that both $\Omega(X)$ and $\mathcal{K}(X)$ are again stably compact when equipped with their Scott-topologies.

For morphisms, there is some choice. The first to come to mind are, of course, the topologically continuous functions, which give rise to the category SCS. However, we prefer to work in SCS* where the morphisms from $X$ to $Y$ are the compact saturated subsets of $X_{\kappa} \times Y$, that is, certain closed relations. Composition is the usual relational product. Note that every relation $R: X \mapsto Y$ can be closed up topologically to yield a morphism in SCS* but this process, in general, is not functorial.

For a continuous function $f: X \rightarrow Y$ the hypergraph $R_{f}:=\left\{(x, y) \in X_{\kappa} \times Y \mid f(x) \leq_{Y}\right.$ $y\}$ is a closed relation and the assignment $f \mapsto R_{f}$ is a faithful functor SCS $\Rightarrow$ SCS . Hence SCS can be identified with a subcategory of SCS*, which turns out to be co-reflective with co-reflection $\mathcal{K}$ which maps $X$ to $\mathcal{K}(X)$ and $R: X \mapsto Y$ to $\left\{\left(K, K^{\prime}\right) \in \mathcal{K}(X) \times \mathcal{K}(Y)\right.$ $\left.K^{\prime}=[K] R\right\}$.

We will also consider $\mathrm{SCS}^{\mathrm{p}}$ where morphisms are (hypergraphs of) functions which are continuous with respect to both the original and the co-compact topology. These are known as perfect maps. SCS* is order enriched if we consider reversed inclusion between the graphs of closed relations. It then turns out that a relation is a perfect function if and only if it is an upper adjoint.

In previous work [JKM99, Keg99, JKM01] we have shown that SCS* enjoys a number of closure properties, to wit, disjoint union (product and coproduct in SCS*), cartesian product (product in SCS), relation space (Kleisli exponential in SCS*), lifting, and bilimits. The purpose of the present note is to discuss the closure under the probabilistic powerspace construction.

Proposition 2.2 ([AMJK]). For a stably compact space $X$, the set $V^{1}(X)$ of probability valuations equipped with the weak topology, is stably compact.

Here, the weak topology is generated by sets of the form $O_{p}:=\left\{v \in V^{1}(X) \mid v(O)>p\right\}$, where $O \in \Omega(X)$ and $0<p<1$. For a closed relation $R: X \mapsto Y$ it is natural to set $v V^{1}(R) v^{\prime} \quad: \Longleftrightarrow \quad \forall U \in \Omega(Y) \cdot v\left(R^{-1}[U]\right) \leq v^{\prime}(U)^{1}$. One observes:

Proposition 2.3. In general, $V^{1}(-)$ does not preserve composition in SCS*. It is, however, a functor from SCS to SCS, which furthermore restricts and corestricts to SCS ${ }^{p}$.

\footnotetext{
${ }^{1}$ For a closed relation $R: X \mapsto Y$ we set $R^{-1}(U):=\{x \in X \mid \forall y \in Y . x R y \Longrightarrow y \in U\}$.
} 


\section{The Multilingual Sequent Calculus}

In this section we review the basic ideas of [JKM99], where the category of multilingual sequent calculi (MLS) was first introduced. An algebra for two binary operations and two constants is called a token algebra. For example, any lattice $(L ; \wedge, \top, \vee, \perp)$ is a token algebra, as is the appropriate term algebra $T(G)$ generated from a set $G$. For two token algebras $L$ and $M$, a consequence relation from $L$ to $M$ is a relation $\vdash \subseteq \mathfrak{P}_{\mathrm{fin}}(L) \times \mathfrak{P}_{\mathrm{fin}}(M)$ obeying Gentzen's rules of positive sequent calculus:

$$
\begin{array}{cc}
\frac{\Gamma \vdash \Delta}{\perp \vdash}(L \perp) & \frac{\overline{\Gamma \vdash \Delta, \perp}}{\Gamma \vdash}(R \perp) \\
\frac{\Gamma \vdash \Delta}{\overline{\top, \Gamma \vdash \Delta}}(L \top) & \frac{\Gamma \top}{\vdash \top}(R) \\
\frac{\phi, \psi, \Gamma \vdash \Delta}{\overline{\phi \wedge \psi, \Gamma \vdash \Delta}}(L \wedge) & \frac{\Gamma \vdash \Delta, \phi \quad \Gamma \vdash \Delta, \psi}{\Gamma \vdash \Delta, \phi \wedge \psi}(R \wedge) \\
\frac{\phi, \Gamma \vdash \Delta \quad \psi, \Gamma \vdash \Delta}{\phi \vee \psi, \Gamma \vdash \Delta}(L \vee) & \frac{\Gamma \vdash \Delta, \phi, \psi}{\overline{\Gamma \vdash \Delta, \phi \vee \psi}}(R \vee) \\
\frac{\Gamma \vdash \Delta}{\Gamma^{\prime}, \Gamma \vdash \Delta, \Delta^{\prime}}(\mathrm{W})
\end{array}
$$

The double lines in the above figures indicate that the rule applies in both directions. This differs from the usual presentation of a sequent calculus in two important ways. First, the tokens (formulas) on either side of a sequent are drawn from different sets. This immediately precludes closing under (Cut), and from including the usual identity axioms: $\phi \vdash \phi$. Second, in proof theory one typically only requires closure under forward application of the rules. However, in the presence of identity axioms and the (Cut) rule, such a relation is in fact also closed under backward application. Because we do not assume either identity axioms or closure under (Cut), we make the closure under backward application explicit. A third, less important difference, is that we allow token algebras to be non-free. As it happens, this is just a convenience as the category MLS is equivalent to its full subcategory consisting of objects defined on free token algebras (which we will examine in the next section). Consequence relations are the morphisms of the category MLS. Composition is defined by the following impoverished version of Gentzen's Cut rule. Given two consequence relations $\vdash: L \rightarrow M$ and $\vdash^{\prime}: M \rightarrow N$, define $\vdash ; \vdash^{\prime}$ by the rule:

$$
\frac{\Gamma \vdash \phi \quad \phi \vdash^{\prime} \Lambda}{\Gamma \vdash ; \vdash^{\prime} \Lambda} \text { (S-Cut) }
$$

This composition is associative, and consequence relations are closed under it. In case domain and target algebra are the same, one can consider Gentzen's original rule:

$$
\frac{\Gamma \Vdash \Delta, \phi \quad \phi, \Theta \Vdash^{\prime} \Lambda}{\Gamma, \Theta \Vdash \circ \Vdash^{\prime} \Delta, \Lambda} \text { (Cut) }
$$


We employ it to define the objects (or, rather, identities) of our category. A continuous sequent calculus on $L$ is a consequence relation $\Vdash_{L}$ from $L$ to $L$ satisfying $\Vdash_{L}=\Vdash_{L} \circ \Vdash_{L}$. (Note that we distinguish notationally between composition by (S-Cut) and (Cut), and between general and endo-relations.) We are now ready to define the category MLS: An object of the category MLS is a token algebra equipped with a continuous sequent calculus $L=\left(L, \Vdash_{L}\right)$. A morphism from $L$ to $M$ is a consequence relation $\vdash: L \rightarrow M$ that is compatible with $L$ and $M$ :

$$
\Vdash_{L} ; \vdash=\vdash=\vdash ; \Vdash_{M}
$$

This leads to the major result of [JKM99]:

Theorem 3.1. The categories MLS and SCS* are equivalent.

In one direction, the isomorphism is given by spec: MLS $\Rightarrow \mathrm{SCS}^{*}$, which assigns to a continuous sequent calculus the set of prime round filters, topologized in the usual way. We describe the inverse at the beginning of Section 5.

Like SCS*, MLS is order-enriched (by inclusion between graphs). The equivalence preserves this enrichment and hence it restricts and corestricts to $\mathrm{SCS}^{\mathrm{p}}$ and $\mathrm{MLS}^{\mathrm{u}}$, the category of upper adjoint consequence relations. We will exhibit a general method for defining adjoints in MLS below.

Closed relations which are hypergraphs of general continuous functions can also be characterized on the MLS side, see [Keg99, Thm 3.1.44].

\section{Free token algebras}

In Logic, formulas are normally built up freely from a set of atomic propositions. The analogous situation for a token algebra $L$ is given when $L$ is the free term algebra $T(G)$ over a set of generators $G$. We will now explore how far the concepts of the multilingual sequent calculus can be expressed solely in terms of generators. This will provide us with the basic toolkit for doing domain constructions in a proof-theoretic fashion. First we note that consequence relations are completely determined by their behavior on generators.

Lemma 4.1 ([Keg99]). Let $L=T(G)$ and $M=T(H)$ be free token algebras and $R \subseteq$ $\mathfrak{P}_{\text {fin }}(G) \times \mathfrak{P}_{\text {fin }}(H)$ be a relation. Denote with $R^{w}$ the closure of $R$ under weakening with generators and $R^{+}$the further closure under the forward logical rules.

1. $R^{+}$is a consequence relation.

2. $R^{+}$, when restricted to generators, equals $R^{w}$.

3. For an arbitrary consequence relation $\vdash$ from $L$ to $M, \vdash=R^{+}$where $R$ is the restriction of $\vdash$ to generators.

In general, a cut formula can not be restricted to generators but with the following slight generalization we do succeed. For a set $G$, define a diagonal pair on $G$ to be a pair $\left\langle\left\{C_{i}\right\}_{i},\left\{D_{j}\right\}_{j}\right\rangle$, both sets of subsets of $G$, provided that for each choice function $f \in \prod_{i} C_{i}$ and choice function $g \in \prod_{j} D_{j}$, there exists $i$ and $j$ so that $f(i)=g(j)$. Given two consequence relations 
$\vdash: L \rightarrow T(G)$ and $\vdash^{\prime}: T(G) \rightarrow N$, define $\vdash ; \vdash^{\prime}$ by the rule:

$$
\begin{array}{cc}
\Gamma \vdash \Delta_{1} & \Theta_{1} \vdash^{\prime} \Lambda \\
\vdots & \vdots \\
\Gamma \vdash \Delta_{m} & \Theta_{n} \vdash^{\prime} \Lambda \\
\hline \Gamma\left(\vdash ; \vdash^{\prime}\right) \Lambda & \left(\text { Cut }^{*}\right)
\end{array}
$$

subject to the condition that $\left\langle\left\{\Delta_{i}\right\}_{i=1}^{m},\left\{\Theta_{j}\right\}_{j=1}^{n}\right\rangle$ is a diagonal pair on $G$.

The following justifies re-using ";" for composition:

Lemma 4.2 ([Keg99]). In the presence of the logical rules, (S-Cut) and (Cut*) are interdefinable.

For the identities we need to simulate the stronger requirement of idempotence with respect to (Cut).

Lemma 4.3. For a consequence relation $\Vdash$ on a free token algebra $L=T(G)$ the following are equivalent.

1. $\Vdash=\Vdash \circ \Vdash$.

2. $\Vdash ; \Vdash \subseteq \Vdash$, and $[\mathrm{L}-\mathrm{Int}]$ and $[\mathrm{R}$-Int $]$ where

[L-Int] If $\phi, \Gamma \Vdash \Delta$, then there exists a diagonal pair $\left\langle\left\{\Lambda_{i}\right\}_{i},\left\{\Theta_{j}\right\}_{j}\right\rangle$ in $G$ so that $\phi \Vdash \Lambda_{i}$ holds for each $i$, and $\Theta_{j}, \Gamma \Vdash \Delta$ holds for each $j$.

[R-Int] If $\Gamma \Vdash \Delta, \psi$, then there exists a diagonal pair $\left\langle\left\{\Lambda_{i}\right\}_{i},\left\{\Theta_{j}\right\}_{j}\right\rangle$ in $G$ so that $\Gamma \Vdash \Delta, \Lambda_{i}$ holds for each $i$, and $\Theta_{j} \Vdash \psi$ holds for each $j$.

Employing free token algebras, our general strategy for defining functors $F: \mathcal{A} \Rightarrow$ MLS will be the following:

1. [Basic tokens] For object $A$, define a set $G_{F}(A)$ and let the token algebra $F(A)$ be the term algebra $T\left(G_{F}(A)\right)$ over $G_{F}(A)$.

2. [Proof rules] For a morphism $f: A \rightarrow B$, define $F^{0}(f)$ to be a relation from finite subsets of $G_{F}(A)$ to finite subsets of $G_{F}(B)$, and let $F(f)$ be $\left(F^{0}(f)\right)^{+}$.

3. [Composition] Show that $F(g \circ f)=F(f) ; F(g)$. Because $F(-)$ is determined by its restriction to generators, this reduces to

(a) [(Cut*) elimination] $F^{0}(f) ; F^{0}(g) \subseteq\left[F^{0}(g \circ f)\right]^{w}$; and

(b) $\left[(\right.$ Cut*) introduction $] F^{0}(g \circ f) \subseteq\left[F^{0}(f) ; F^{0}(g)\right]^{w}$.

4. [Identities] Show that $F$ preserves identities. In light of [(Cut*) elimination] above, this reduces to $[L-\operatorname{lnt}]$ and $[R-I n t]$.

We label step (2) [Proof rules] because $F^{0}(f)$ can typically be presented in the form:

$$
\frac{P(f: A \rightarrow B, \Gamma, \Delta)}{\Gamma F(f) \Delta}(F)
$$


where $P$ is some predicate on morphisms of $\mathcal{A}$ and finite sets of generators. The first two steps of the method are purely formal. The third and fourth steps constitute the verification that we have defined a functor. Also note that the conditions [(Cut*) introduction], [L-Int] and [R-Int] are quite natural in traditional proof theory. They amount to the requirement that derivable sequents can always arise as the result of (Cut*) of a specific form. This sort of meta-theorem is used, for example, to derive the Craig Interpolation Theorem: If $\Gamma \Rightarrow \Delta$, then there is a formula $\phi$ involving only non-logical symbols occurring in both $\Gamma$ and $\Delta$ so that $\Gamma \Rightarrow \phi$ and $\phi \Rightarrow \Delta$. Thus the conditions on functors amount to a formalization of "good" behavior for constructions in the logic MLS.

Our principle tool for showing that two objects of MLS are isomorphic is the following.

Lemma 4.4. Suppose $L$ and $M$ are continuous sequent calculi and $h: M \rightarrow L$ is a map between the underlying token algebras. Consider the following properties

[hom] h is a homomorphism.

[smooth] Whenever $\Gamma \Vdash_{L} h(\phi)$ then there exists $\phi^{\prime} \in M$ such that $\phi^{\prime} \Vdash_{M} \phi$ and $\Gamma \Vdash_{L} h\left(\phi^{\prime}\right)$. Likewise, with $h(\phi) \Vdash_{L} \Gamma$ we have $\phi \Vdash_{M} \phi^{\prime}$ such that $h\left(\phi^{\prime}\right) \Vdash_{L} \Gamma$.

[I--preserving] $\Delta \Vdash_{M} \Delta^{\prime}$ implies $h(\Delta) \Vdash_{L} h\left(\Delta^{\prime}\right)$ (where $h(\Delta)$ is short for $h\left(\psi_{1}\right), \ldots, h\left(\psi_{n}\right)$ whenever $\left.\Delta=\psi_{1}, \ldots, \psi_{n}\right)$.

$\left[\Vdash-\right.$-reflecting $h(\Delta) \Vdash_{L} h\left(\Delta^{\prime}\right)$ implies $\Delta \Vdash_{M} \Delta^{\prime}$.

[dense] $\Gamma \Vdash_{L} \Gamma^{\prime}$ implies that there exists $\phi \in M$ with $\Gamma \Vdash_{L} h(\phi) \Vdash_{L} \Gamma^{\prime}$.

Define relations $\vdash^{h} \subseteq \mathfrak{P}_{\text {fin }}(L) \times \mathfrak{P}_{\text {fin }}(M)$ and $\vdash_{h} \subseteq \mathfrak{P}_{\text {fin }}(M) \times \mathfrak{P}_{\text {fin }}(L)$ by setting

$$
\Gamma \vdash^{h} \Delta \text { if } \Gamma \Vdash_{L} h(\Delta) \text {, and } \Delta \vdash_{h} \Gamma \text { if } h(\Delta) \Vdash_{L} \Gamma \text {. }
$$

1. If $h$ is a smooth homomorphism then $\vdash^{h}$ and $\vdash_{h}$ are compatible consequence relations.

2. If $h$ is a smooth homomorphism which is also $\Vdash$-preserving then $\vdash^{h}$ is the upper adjoint to $\vdash_{h}$. That is, $\left(\vdash^{h} ; \vdash_{h}\right) \subseteq \vdash_{L}$ and $\vdash_{M} \subseteq\left(\vdash_{h} ; \vdash^{h}\right)$.

3. If $h$ is a smooth homomorphism which is also $\Vdash$-reflecting then $\left(\vdash_{h} ; \vdash^{h}\right) \subseteq \vdash_{M}$.

4. If $h$ is a smooth homomorphism which is also dense then $\vdash_{L} \subseteq\left(\vdash^{h} ; \vdash_{h}\right)$.

We observe that in the presence of $\Vdash$-preservation, the homomorphism condition is not needed. In practice, however, $M$ is often a free token algebra $T(G)$ and $h$ is defined as the homomorphic extension of a map from $G$ to $L$. In this situation it is sufficient to check smoothness, $\Vdash$-preservation and reflection for lists $\Delta$ of generators only.

Also note that in the presence of $\Vdash$-reflection, smoothness is subsumed by density. With these two observations, the following extension from objects to functors becomes a straightforward corollary.

Lemma 4.5. Suppose $F: \mathcal{A} \Rightarrow \mathrm{MLS}$ and $G: \mathcal{A} \Rightarrow$ MLS are functors, and for each object $A \in \mathcal{A}, h_{A}: G(A) \rightarrow F(A)$ is a dense map between token algebras. If for each $f: A \rightarrow B$ in $\mathcal{A}$,

$$
\Gamma G(f) \Delta \text { if and only if } h_{A}(\Gamma) F(f) h_{B}(\Delta)
$$

then $\vdash^{h_{A}}$ is a natural isomorphism from $F$ to $G$ with inverse $\vdash_{h_{A}}$. 


\section{Domain constructions in logical form}

We will now illustrate how the general techniques of the previous section can be used for proving that an endofunctor functor $\bar{F}$ in MLS is a logical description of an endofunctor $F$ in SCS*. We start by defining a functor lang from SCS* to MLS (which is in fact one half of the equivalence stated in Theorem 3.1). We set

$$
G_{\text {lang }}(X):=\{(O, K) \in \Omega(X) \times \mathcal{K}(X) \mid O \subseteq K\}
$$

and let lang $(X)$ be the free term algebra over these generators. For each closed relation $R: X \rightarrow Y$, define $\vdash_{R}=$ lang $(R)$ by the rule:

$$
\frac{\left[\bigcap_{i=1}^{m} K_{i}\right] R \subseteq \bigcup_{j=1}^{n} O_{j}^{\prime}}{\left(O_{1}, K_{1}\right), \ldots,\left(O_{m}, K_{m}\right) \vdash_{R}\left(O_{1}^{\prime}, K_{1}^{\prime}\right), \ldots,\left(O_{n}^{\prime}, K_{n}^{\prime}\right)} \text { (lang) }
$$

We refer the reader to [JKM99] for the proof that spec and lang determine an equivalence.

By a construction over spaces we mean a functor $\mathrm{T}: \mathrm{SCS}^{*} \Rightarrow \mathrm{SCS}^{*}$. We seek to find an analogue $\overline{\mathrm{T}}$ on the side of MLS, that is, we wish to show that the two functors lang $\circ$ $\mathrm{T}$ and $\overline{\mathrm{T}} \circ$ lang are naturally isomorphic. For this we will employ the general technique described in the previous section, adapted to this special situation. Consider the objects first: Because SCS* and MLS are isomorphic categories, we can replace lang $(X)$ by an isomorphic "concrete" sequent calculus $L$, where the isomorphism is witnessed in the style of Lemma 4.4. To wit, we assume that we are given a map $\llbracket-\rrbracket: L \rightarrow \operatorname{lang}(X)$, consisting of components $o_{L} \llbracket-\rrbracket: L \rightarrow \Omega(X)$ and $\kappa_{L} \llbracket-\rrbracket: L \rightarrow \mathcal{K}(X)$ such that

- $\forall \phi \in L . o_{L} \llbracket \phi \rrbracket \subseteq \kappa_{L} \llbracket \phi \rrbracket ;$

- $\forall \phi, \psi \in L . \phi \Vdash_{L} \psi$ if and only if $\kappa_{L} \llbracket \phi \rrbracket \subseteq o_{L} \llbracket \psi \rrbracket$;

- $\forall K \in \mathcal{K}(X), O \in \Omega(X) . K \subseteq O \Longrightarrow \exists \phi \in L . K \subseteq o_{L} \llbracket \phi \rrbracket$ and $\kappa_{L} \llbracket \phi \rrbracket \subseteq O$.

The task, then, is to define a sequent calculus $\overline{\mathrm{T}}(L)$ isomorphic to lang $\circ \mathrm{T}(X)$. We will do this by exhibiting a set of generators $G_{L}$ for $\overline{\mathrm{T}}(L)$ together with interpretations $o_{\overline{\mathrm{T}}(L)} \llbracket-\rrbracket: G_{L} \rightarrow$ $\Omega(\mathrm{T}(X))$ and $\kappa_{\overline{\mathrm{T}}(L)} \llbracket-\rrbracket: G_{L} \rightarrow \mathcal{K}(\mathrm{T}(X))$ such that the three conditions above are again satisfied.

For morphisms, the task is almost the same. We assume maps $o_{L} \llbracket-\rrbracket, \kappa_{L} \llbracket-\rrbracket$ and $o_{K} \llbracket-\rrbracket$, $\kappa_{K} \llbracket-\rrbracket$ which witness the isomorphism between $L$ and $\operatorname{lang}(X)$, and $M$ and lang $(Y)$, respectively. We also assume that the compatible consequence relation $\vdash: L \rightarrow M$ represents the SCS* relation $R: X \rightarrow Y$ in the sense that

- $\forall \phi \in L, \psi \in M . \phi \vdash \psi$ if and only if $\left[\kappa_{L} \llbracket \phi \rrbracket\right] \subseteq o_{M} \llbracket \psi \rrbracket$.

This property must be preserved by the spatial and the logical construction:

- $\forall \Gamma \subseteq G_{L}, \Delta \subseteq G_{M} . \Gamma \mathrm{T}(\vdash) \Delta$ if and only if $\left[\bigcap_{\phi \in \Gamma} \kappa_{\overline{\overline{\mathrm{T}}(L)}} \llbracket \phi \rrbracket\right] \mathrm{T}(R) \subseteq \bigcup_{\psi \in \Delta} o_{\overline{\overline{\mathrm{T}}(M)}} \llbracket \psi \rrbracket$. 


\section{The probabilistic powerspace construction}

We are now ready to embark on our logical characterisation of the probabilistic powerspace of a stably compact space. Since a direct proof, despite the tools above, is still too complicated, we perform the construction in four stages, starting with the function space $\mathrm{C} \Omega(X)=$ $[\Omega(X) \rightarrow[0,1]]$. This follows the strategy in [Hec94].

We first observe that because both $\Omega(X)$ and $[0,1]$ are continuous lattices, $\mathrm{C} \Omega(X)$ is also a continuous lattice and therefore stably compact in its Scott-topology. The latter coincides with the weak topology generated by sets of the form

$$
O_{p}:=\{v \in \mathbf{C} \Omega(X) \mid v(O)>p\}
$$

We therefore choose as generators for $\mathrm{C} \Omega(L)$ tokens $\phi_{p}$ where $\phi \in L$ and $0<p<1$ with the following interpretation function for open sets:

$$
o_{\mathrm{C} \Omega} \llbracket \phi_{p} \rrbracket:=\left\{v \in \mathrm{C} \Omega(X) \mid v\left(o_{L} \llbracket \phi \rrbracket\right)>p\right\}
$$

For the compact interpretation we define $\bar{v}: \mathcal{K}(X) \rightarrow[0,1]$ by

$$
\bar{v}(K):=\inf \{v(U) \mid U \supseteq K\}
$$

and set

$$
\kappa_{\mathrm{C} \Omega} \llbracket \phi_{p} \rrbracket:=\left\{v \in \mathrm{C} \Omega(X) \mid \bar{v}\left(\kappa_{L} \llbracket \phi \rrbracket\right) \geq p\right\}
$$

The consequence relation on $\mathrm{C} \Omega(L)$ is generated by the single proof rule

$$
\frac{\phi \Vdash_{L} \psi \quad p>q}{\phi_{p} \Vdash_{\mathrm{C} \Omega} \psi_{q}}(\mathrm{C} \Omega)
$$

Using the general technique outlined in the previous section, it is now not too hard to show that this indeed is a logical description of $\mathrm{C} \Omega(X)$ :

Proposition 6.1. $\overline{\mathrm{C} \Omega}(L)$ and lang $(\mathrm{C} \Omega(X))$ are isomorphic.

The extension to morphisms is straightforward:

$$
\frac{\phi \vdash \psi \quad p>q}{\phi_{p} \mathrm{C} \Omega(\vdash) \psi_{q}}(\mathrm{C} \Omega)
$$

and together with the previous proposition this yields:

Theorem 6.2. The functor $\overline{\mathrm{C} \Omega} \circ$ lang is naturally isomorphic to lang $\circ \mathrm{C} \Omega$, in other words, $\overline{\mathrm{C} \Omega}: \mathrm{MLS} \Rightarrow \mathrm{MLS}$ is a logical description of the construction $\mathrm{C} \Omega: \mathrm{SCS}^{*} \Rightarrow \mathrm{SCS}^{*}$.

We refine the isomorphism established in the preceding Theorem by restricting the construction to more specialized function spaces. Let us first consider the general situation. Suppose already have a logical description $L$ of a space $X$ and seek a logical description for a subspace $Y \subseteq X$. The idea is to keep the token algebra $L$ but to strengthen the internal reasoning $\Vdash$ with additional proof rules, resulting in a consequence relation $\Vdash^{\prime}$. This is in analogy to locale theory where a sublocale is defined as a congruence on the frame. In our setting, 
we intend to use Lemma 4.4 with $h$ being the identity on $L$. It is then immediate that (hom) and (I--preservation) are satisfied, and that ( $\Vdash$-reflection) cannot hold unless $Y=X$. What needs to be shown is smoothness and density, which can be expressed as $\Vdash \vdash^{\prime} \Vdash^{\prime}=\Vdash^{\prime}=\Vdash^{\prime}$; $\vdash^{\prime}$. Since $\Vdash^{\prime}$ is given by an additional proof rule, the inclusions $\Vdash ; \Vdash^{\prime} \subseteq \Vdash^{\prime}$ and $\Vdash^{\prime} ; \Vdash \subseteq \Vdash^{\prime}$ hold by convention, and it all boils down to showing the other directions. In the situation at hand, this will not be difficult.

Once this work is done, we conclude from Lemma 4.4 that $\operatorname{spec}\left(L, \Vdash^{\prime}\right)$ is a perfect subspace of $\operatorname{spec}(L, \Vdash) \cong X$, and it remains to show that this subspace is indeed the desired $Y$. To this end, one shows that for $x \in X$, the neighborhood filter is closed under the new proof rule if and only if $x \in Y$. This will complete the argument.

To restrict to those functions in $\mathrm{C} \Omega(X)$ which assign 0 to the empty set, we add the rule

$$
\overline{\perp_{p} \Vdash}(\mathrm{Str})
$$

The resulting construction is still functorial on all of SCS* and MLS, respectively.

For modularity, note that our tokens stipulate lower bounds only. So we must break modularity into its constituent inequalities. Say that $v: \Omega(X) \rightarrow[0,1]$ is sub-modular if

$$
v(U)+v(V) \leq v(U \cup V)+v(U \cap V)
$$

and that $v$ is super-modular if

$$
v(U)+v(V) \geq v(U \cup V)+v(U \cap V)
$$

These two properties are characterised by the following proof rules. For sub-modularity add:

$$
\frac{\phi \Vdash_{L} \rho \quad \psi \Vdash_{L} \rho \quad \phi, \psi \Vdash_{L} \sigma \quad p+q>r+s}{\phi_{p}, \psi_{q} \Vdash_{\mathrm{V}(L)} \rho_{r}, \sigma_{s}} \text { (Sub-mod) }
$$

and for super-modularity add:

$$
\frac{\phi \Vdash_{L} \rho \quad \phi \Vdash_{L} \sigma \quad \psi \Vdash_{L} \rho, \sigma \quad p+q>r+s}{\phi_{p}, \psi_{q} \Vdash_{\mathrm{V}(L)} \rho_{r}, \sigma_{s}} \text { (Super-mod) }
$$

We note that the resulting construction $V$ is functorial only for SCS and MLS ${ }^{\dagger}$, respectively. This restriction is not too surprising because SCS* is the Kleisli category of SCS with respect to the monad $\mathcal{K}$, which on domains is known to be the Smyth-powerdomain [AJ94, Thm 6.2.14]. Having $V$ functorial on SCS* would therefore amount to a combination of nondeterminism and probabilistic choice. It has become clear recently that this problem cannot have a simple solution because there is no distributive law between these two constructions. We refer the reader to [Mis00, Tix99, Var02] for a more detailed discussion.

To complete our construction we consider the condition $v(X)=1$ for normal valuations. In $L, o \llbracket \phi \rrbracket=X$ if and only if $\vdash_{L} \phi$ (if and only if $\phi$ is logically equivalent to $T$ with respect to $\left.\vdash_{L}\right)$. So $\vdash_{\mathrm{V}(L)}$ restricts further to normal valuations by adding the rule:

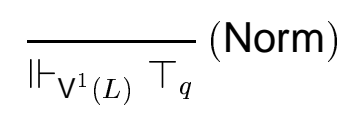

All rules necessary to characterize $\mathrm{V}^{1}(X)$ are collected together in Figure 1.

We conclude by stating a result which is shown with very different methods than the ones employed in the present note, and which we cannot fully spell out for lack of space:

Theorem 6.3. Assuming the continuous sequent calculus $L$ is decidable, then so is $V^{1}(L)$. 


$$
\begin{aligned}
& \frac{\phi \Vdash_{L} \psi \quad p>q}{\phi_{p} \Vdash \psi_{q}}(\mathrm{C} \Omega) \\
& \overline{\perp_{p} \Vdash}\left(\mathrm{Str}^{\prime}\right) \quad \frac{}{\Vdash \top_{q}}(\text { Norm }) \\
& \frac{\phi \Vdash_{L} \rho \quad \psi \Vdash_{L} \rho \quad \phi, \psi \Vdash_{L} \sigma \quad p+q>r+s}{\phi_{p}, \psi_{q} \Vdash \rho_{r}, \sigma_{s}} \text { (Sub-mod) } \\
& \frac{\phi \Vdash_{L} \rho \quad \phi \Vdash_{L} \sigma \quad \psi \Vdash_{L} \rho, \sigma \quad p+q>r+s}{\phi_{p}, \psi_{q} \Vdash \rho_{r}, \sigma_{s}} \text { (Super-mod) }
\end{aligned}
$$

continuous sequent calculus $\overline{\mathrm{V}}^{1}(L)$.]

Figure 1: The proof rules for Probabilistic Domain Logic.

\section{Conclusions and further work}

The papers [JKM99, Keg99, JKM01] and the present note confirm, in our opinion, that the category SCS* offers a flexible and convenient universe of semantic spaces. As we have emphasized all along, one of its key features is its intimate relationship with (very standard!) logic via Stone duality. This allows us to describe spaces and constructions spatially, localically, and logically in a straightforward and elegant fashion.

Trying to establish the equivalence of logical and spatial domain constructions on the logical side has shown that this requires concepts and techniques from Proof Theory such as cut elimination and interpolation, a connection which has hitherto - to the best of our knowledge - not been observed.

SCS* strictly extends all common classes of algebraic and continuous domains, and contains classical spaces such as the unit interval in its Hausdorff topology. The probabilistic powerdomain shows that this extension is necessary, as there is no other suitably closed category available to us which accommodates this construction. The modularity axioms of our logical characterisation of the probabilistic powerdomain also demonstrate that the extension of domain logic to full (rather than intuitionistic) sequents is advantageous.

As a semantic universe, SCS* takes the notion of a non-deterministic (rather than functional) computation as basic, which is, of course, reminiscent of traditional work in programming languages [Dij76], but which has also more recently been found to be fundamental to exact real number computation [Lon99]. This provides an exciting prospect for future work.

In previous work, [Jon90, Hec96, Tix99], the probabilistic powerdomain has been characterised as a free cone over the space $X$. It is would be interesting to see if this characterization can be used to prove completeness of our axiomatization without referring to the spatial side at all. Such an approach was carried out successfully in [Keg99] for the more "categorical" 
constructions on SCS*.

Having laid the groundwork, it should now be possible to establish the precise connection to work in probabilistic verification. More speculatively, perhaps, one could also try to extend the present work so as to capture more accurately truly observable properties of probabilistic programs, that is, to model the Bayesian view of probability.

\section{Acknowledgements}

The research reported here was started when the first author visited the School of Computer Science of the University of Birmingham in the Summer of 2001, supported by a guest professorship of that department. We have also greatly profited from insightful comments by anonymous referees on an earlier version of this paper.

\section{References}

[Abr90] S. Abramsky, The lazy lambda calculus, Research Topics in Functional Programming (D. Turner, ed.), Addison Wesley, 1990, pp. 65-117.

[Abr91] S. Abramsky, A domain equation for bisimulation, Information and Computation 92 (1991), 161-218.

[AJ94] S. Abramsky and A. Jung, Domain theory, Handbook of Logic in Computer Science (S. Abramsky, D. M. Gabbay, and T. S. E. Maibaum, eds.), vol. 3, Clarendon Press, 1994, pp. 1-168.

[AM01] M. Alvarez-Manilla, Measure theoretic results for continuous valuations on partially ordered spaces, Ph.D. thesis, Imperial College, University of London, 2001.

[AMESD00] M. Alvarez-Manilla, A. Edalat, and N. Saheb-Djahromi, An extension result for continuous valuations, Journal of the London Mathematical Society 61 (2000), 629-640.

[AMJK] M. Alvarez-Manilla, A. Jung, and K. Keimel, Valuations on a stably compact space, In preparation.

[DEP97] Josee Desharnais, Abbas Edalat, and Prakash Panangaden, Bisimulation for labelled markov processes, Proceedings of the 12th IEEE Symposium on Logic in Computer Science, 1997, pp. 149-158.

[DEP98] Josee Desharnais, Abbas Edalat, and Prakash Panangaden, A logical characterization of bisimulation for labeled markov processes, Logic in Computer Science, 1998, pp. 478487.

[DEPar] J. Desharnais, A. Edalat, and P. Panangaden, Bisimulation for labelled Markov processes, Information and Computation (to appear).

[Dij76] E. W. Dijkstra, A Discipline of Programming, Prentice-Hall, Englewood Cliffs, New Jersey, 1976.

[Eda95] A. Edalat, Dynamical systems, measures and fractals via domain theory, Information and Computation 120 (1995), no. 1, 32-48. 
$\left[\mathrm{GHK}^{+} 80\right] \quad$ G. Gierz, K. H. Hofmann, K. Keimel, J. D. Lawson, M. Mislove, and D. S. Scott, A compendium of continuous lattices, Springer Verlag, 1980.

$\left[\mathrm{GHK}^{+}\right.$02] G. Gierz, K. H. Hofmann, K. Keimel, J. D. Lawson, M. Mislove, and D. S. Scott, Continuous lattices and domains, Cambridge University Press, 2002, Revised edition of $\left[\mathrm{GHK}^{+} 80\right]$, forthcoming.

[Hec94] Reinhold Heckmann, Probabilistic power domains, information systems, and locales, Mathematical Foundations of Programming Semantics VIII (S. Brookes, M. Main, A. Melton, M. Mislove, and D. Schmidt, eds.), 1994, In LNCS 802:1994, pp. 410-437.

[Hec96] R. Heckmann, Spaces of valuations, Papers on General Topology and Applications: Eleventh Summer Conference at the University of Southern Maine (S. Andima, R. C. Flagg, G. Itzkowitz, P. Misra, Y. Kong, and R. Kopperman, eds.), Annals of the New York Academy of Sciences, vol. 806, 1996, pp. 174-200.

[JKM97] A. Jung, M. Kegelmann, and M. A. Moshier, Multi lingual sequent calculus and coherent spaces, 13th Conference on Mathematical Foundations of Programming Semantics (S. Brookes and M. Mislove, eds.), Electronic Notes in Theoretical Computer Science, vol. 6, Elsevier Science Publishers B.V., 1997, 18 pages.

[JKM99] A. Jung, M. Kegelmann, and M. A. Moshier, Multi lingual sequent calculus and coherent spaces, Fundamenta Informaticae 37 (1999), 369-412.

[JKM01] A. Jung, M. Kegelmann, and M. A. Moshier, Stably compact spaces and closed relations, 17th Conference on Mathematical Foundations of Programming Semantics (S. Brookes and M. Mislove, eds.), Electronic Notes in Theoretical Computer Science, vol. 45, Elsevier Science Publishers B.V., 2001, 24 pages.

[Jon90] C. Jones, Probabilistic non-determinism, Ph.D. thesis, University of Edinburgh, Edinburgh, 1990, Also published as Technical Report No. CST-63-90.

[JP89] C. Jones and G. Plotkin, A probabilistic powerdomain of evaluations, Proceedings of the 4th Annual Symposium on Logic in Computer Science, IEEE Computer Society Press, 1989, pp. 186-195.

[JS96] A. Jung and Ph. Sünderhauf, On the duality of compact vs. open, Papers on General Topology and Applications: Eleventh Summer Conference at the University of Southern Maine (S. Andima, R. C. Flagg, G. Itzkowitz, P. Misra, Y. Kong, and R. Kopperman, eds.), Annals of the New York Academy of Sciences, vol. 806, 1996, pp. 214-230.

[JT98] A. Jung and R. Tix, The troublesome probabilistic powerdomain, Proceedings of the Third Workshop on Computation and Approximation (A. Edalat, A. Jung, K. Keimel, and M. Kwiatkowska, eds.), Electronic Notes in Theoretical Computer Science, vol. 13, Elsevier Science Publishers B.V., 1998, 23 pages.

[Keg99] M. Kegelmann, Factorisation systems on domains, Applied Categorical Structures 7 (1999), no. 1-2, 113-128.

[Koz81] D. Kozen, Semantics of probabilistic programs, Journal of Computer and System Sciences 22 (1981), 328-350.

[Law82] J. D. Lawson, Valuations on continuous lattices, Continuous Lattices and Related Topics (Rudolf-Eberhard Hoffmann, ed.), Mathematik Arbeitspapiere, vol. 27, Universität Bremen, 1982, pp. 204-225. 
[Lon99] J.R. Longley, When is a functional program not a functional program?, Proceedings of Fourth ACM SIGPLAN International Conference on Functional Programming, ACM Press, 1999.

[McI01] Annabelle McIver, A generalisation of stationary distributions, and probabilistic program algebra, Electronic Notes in Theoretical Computer Science (Stephen Brookes and Michael Mislove, eds.), vol. 45, Elsevier Science Publishers, 2001.

[Mis00] M. W. Mislove, Nondeterminism and probabilistic choice: Obeying the law, Proceedings 11th CONCUR, Lecture Notes in Computer Science, vol. 1877, Springer Verlag, 2000, pp. 350-364.

[MMS96] Carroll Morgan, Annabelle McIver, and Karen Seidel, Probabilistic predicate transformers, ACM Transactions on Programming Languages and Systems 18 (1996), no. 3, $325-353$.

[SD80] N. Saheb-Djahromi, CPO's of measures for nondeterminism, Theoretical Computer Science 12 (1980), 19-37.

[Smy83] M. B. Smyth, Powerdomains and predicate transformers: a topological view, Automata, Languages and Programming (J. Diaz, ed.), Lecture Notes in Computer Science, vol. 154, Springer Verlag, 1983, pp. 662-675.

[Smy92a] M. B. Smyth, Stable compactification I, Journal of the London Mathematical Society 45 (1992), 321-340.

[Smy92b] M. B. Smyth, Topology, Handbook of Logic in Computer Science, vol. 1 (S. Abramsky, D. M. Gabbay, and T. S. E. Maibaum, eds.), Clarendon Press, 1992, pp. 641-761.

[Tix99] R. Tix, Continuous D-cones: Convexity and powerdomain constructions, Ph.D. thesis, Technische Universität Darmstadt, 1999.

[Var02] D. Varacca, The powerdomain of indexed valuations, 17th LICS conference, IEEE Computer Society Press, 2002.

[Vic89] S. J. Vickers, Topology via logic, Cambridge Tracts in Theoretical Computer Science, vol. 5, Cambridge University Press, 1989. 